\title{
Particle size-resolved source apportionment of primary and secondary organic tracer compounds at urban and rural locations in Spain
}

\author{
B. L. van Drooge and J. O. Grimalt
}

Correspondence to: B. L. van Drooge (barend.vandrooge@idaea.csic.es)

The copyright of individual parts of the supplement might differ from the CC-BY 3.0 licence. 
Table S1. Summary of meteorological conditions in Warm and Cold sampling periods in the Rural and Urban site.

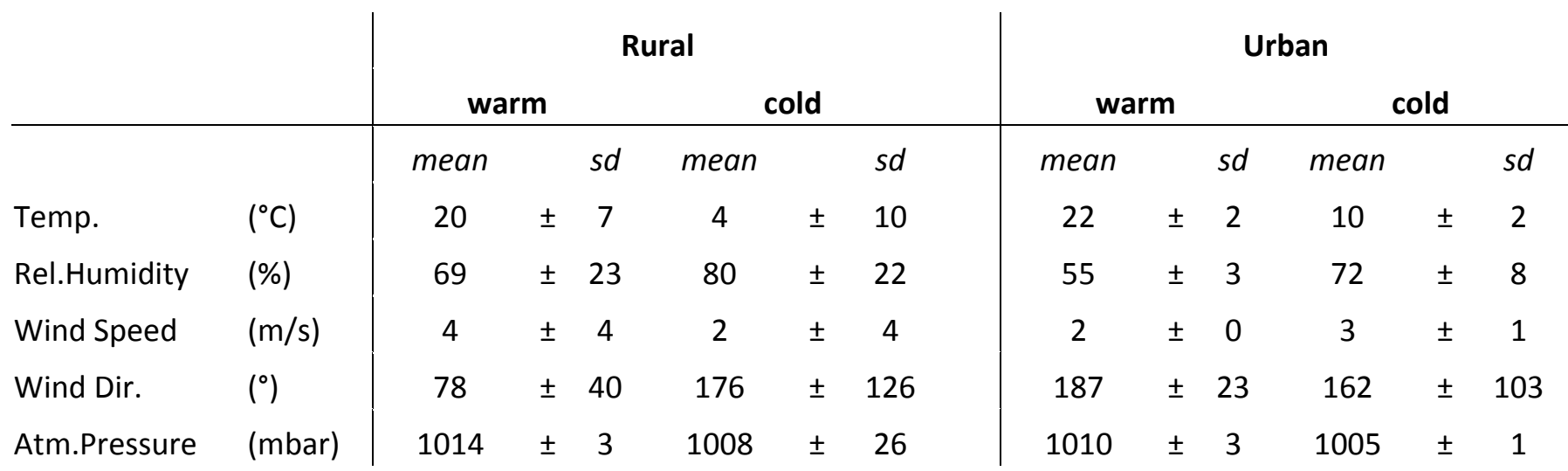


Table S2. Selected ions $(\mathrm{m} / \mathrm{z})$ for the identification and quantification of molecular organic tracer compounds in the PM filter sample extract in GC-MS (EI).

\begin{tabular}{|c|c|c|}
\hline compound family & compound name & ion $m / z$ \\
\hline \multirow{19}{*}{ acids+polyols } & succinic acid (SA) & 247 \\
\hline & glutaric acid (GA) & 261 \\
\hline & adipic acid (AdA) & 275 \\
\hline & pimelic acid (PA) & 289 \\
\hline & suberic acid (SbA) & 303 \\
\hline & azelaic acid (AzA) & 317 \\
\hline & glyceric acid (GyA) & 292 \\
\hline & malic acid (MA) & 233 \\
\hline & 3-hydroxyglutaric acid (3HGA) & 349 \\
\hline & 3-methyl-1,2,3-butanetricarboxylic acid (MBTCA) & 405 \\
\hline & cis-pinonic acid (CPA) & 171 \\
\hline & pinic acid (PNA) & 171 \\
\hline & 2-methylglyceric acid (2MGA) & 219 \\
\hline & C5-alkene triols (C5T) & 231 \\
\hline & 2-methylthreitol (2MT1) & 219 \\
\hline & 2-methylerythritol (2MT2) & 219 \\
\hline & phthalic acid (PhA) & 295 \\
\hline & terephthalic acid (TPhA) & 295 \\
\hline & C16:0 to C21:0 including C18:1 (oleic acid) & 117 \\
\hline \multirow{10}{*}{ saccharides } & galactosan (G) & 217 \\
\hline & mannosan (M) & 204 \\
\hline & levoglucosan (L) & 204 \\
\hline & xylitol (X) & 217 \\
\hline & mannitol (MaOL & 319 \\
\hline & $\alpha$-glucose $(\alpha \mathrm{GL})$ & 204 \\
\hline & $\beta$-glucose $(\beta G L)$ & 204 \\
\hline & dehydrabietic acid (DHA) & 239 \\
\hline & sucrose (S) & 261 \\
\hline & mycose (My) & 261 \\
\hline alkaloid & nicotine (NIC) & 84 \\
\hline \multirow{6}{*}{ quinones } & fluorenone (flo) & 180 \\
\hline & phenanthrenequinone (pheno) & 208 \\
\hline & anthracenequinone (anto) & 208 \\
\hline & benzo[a]fluorenone (baflo) & 230 \\
\hline & benzo[b]fluorenone (bbflo) & 230 \\
\hline & benzanthrenone (bao) & 230 \\
\hline \multirow{15}{*}{ PAH } & phenanthrene (phe) & 178 \\
\hline & anthracene (ant) & 178 \\
\hline & fluoranthene (fla) & 202 \\
\hline & pyrene (pyr) & 202 \\
\hline & retene (ret) & 219 \\
\hline & benz[a]anthracene (baa) & 228 \\
\hline & chrysene (chry) & 228 \\
\hline & benzo[b+j]fluoranthene (bbjfla) & 252 \\
\hline & benzo[k]fluoranthene (bkfla) & 252 \\
\hline & benzo[e]pyrene (bep) & 252 \\
\hline & benzo[a]pyrene (bap) & 252 \\
\hline & indeno[123cd]pyrene (ip) & 276 \\
\hline & dibenz[ah]anthracene (dba) & 278 \\
\hline & benzo[ghi]perylene (bgp) & 276 \\
\hline & coronene (cor) & 300 \\
\hline \multirow{2}{*}{ hopanes } & 17a(H)21ß(H)-29-norhopane (norHop) & 191 \\
\hline & $17 \mathrm{a}(\mathrm{H}) 21 \beta(\mathrm{H})$-hopane (Hop) & 191 \\
\hline n-alkanes & nC20 to nC34 & 71 \\
\hline
\end{tabular}


Table S3a-b. Summary of organic tracer compound concentrations in Warm and Cold sampling periods in the Rural site.

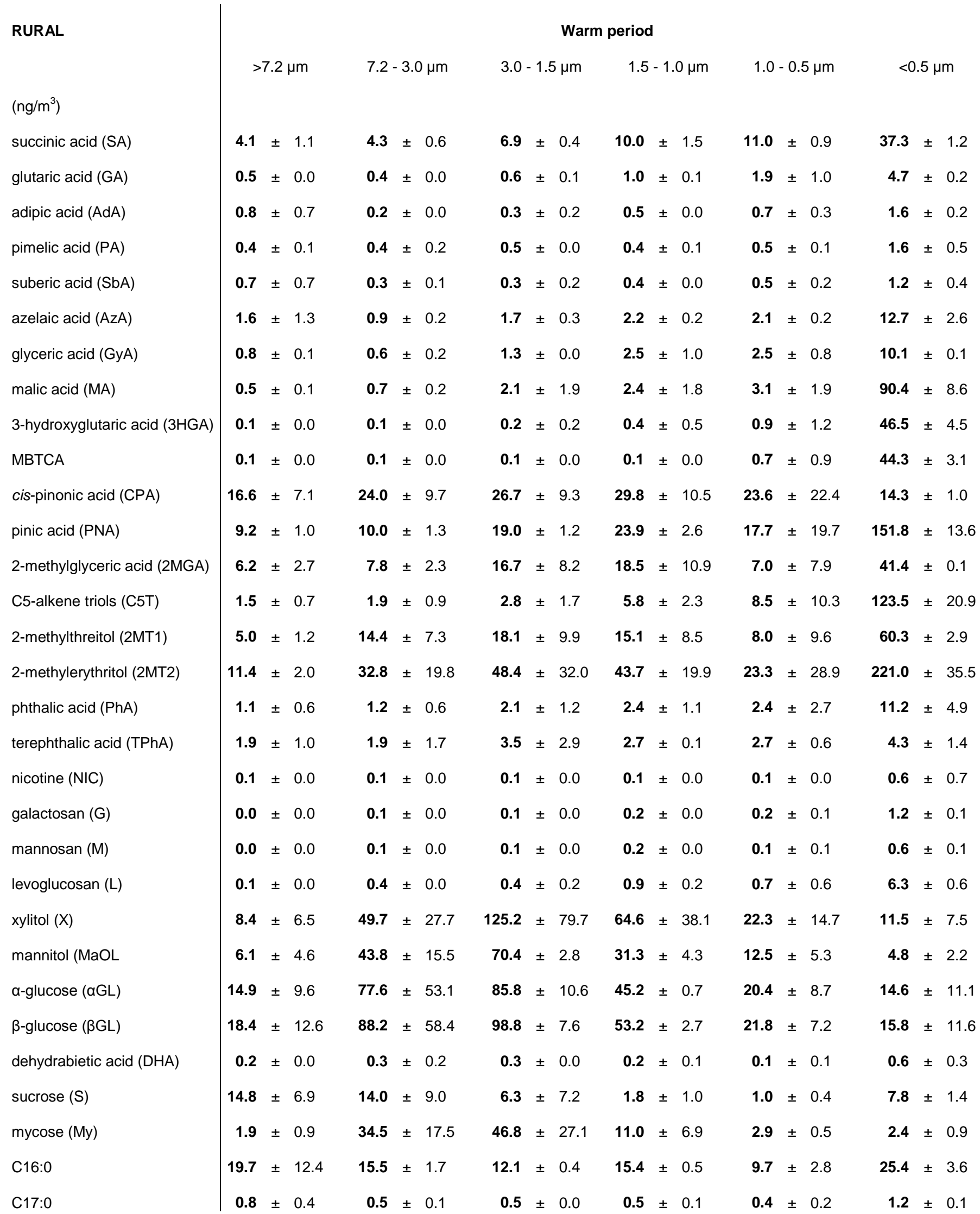




\begin{tabular}{|c|c|c|c|c|c|c|c|c|c|c|c|c|c|c|c|c|c|c|}
\hline C18:1 & 2.6 & \pm & 2.4 & 2.3 & \pm & 0.9 & 1.8 & \pm & 0.5 & 1.2 & \pm & 0.6 & 0.8 & \pm & 0.3 & 1.7 & \pm & 1.4 \\
\hline C18:0 & 4.1 & \pm & 1.8 & 6.1 & \pm & 0.7 & 4.2 & \pm & 0.8 & 3.9 & \pm & 0.0 & 1.9 & \pm & 0.8 & 7.4 & \pm & 2.1 \\
\hline C19:0 & 0.1 & \pm & 0.1 & 0.2 & \pm & 0.0 & 0.2 & \pm & 0.0 & 0.2 & \pm & 0.0 & 0.2 & \pm & 0.2 & 0.5 & \pm & 0.0 \\
\hline C20:0 & 0.4 & \pm & 0.2 & 0.5 & \pm & 0.2 & 0.5 & \pm & 0.1 & 0.4 & \pm & 0.0 & 0.2 & \pm & 0.2 & 1.5 & \pm & 0.0 \\
\hline $\mathrm{C} 21: 0$ & 0.1 & \pm & 0.0 & 0.1 & \pm & 0.1 & 0.2 & \pm & 0.2 & 0.1 & \pm & 0.0 & 0.1 & \pm & 0.0 & 0.5 & \pm & 0.1 \\
\hline fluorenone (flo) & 0.00 & \pm & 0.00 & 0.00 & \pm & 0.00 & 0.00 & \pm & 0.00 & 0.00 & \pm & 0.00 & 0.00 & \pm & 0.00 & 0.01 & \pm & 0.00 \\
\hline phenanthraquinone (pheno) & 0.00 & \pm & 0.00 & 0.00 & \pm & 0.00 & 0.00 & \pm & 0.00 & 0.00 & \pm & 0.00 & 0.00 & \pm & 0.00 & 0.00 & \pm & 0.00 \\
\hline anthracenequinone (anto) & 0.00 & \pm & 0.00 & 0.00 & \pm & 0.00 & 0.00 & \pm & 0.00 & 0.00 & \pm & 0.00 & 0.00 & \pm & 0.00 & 0.03 & \pm & 0.00 \\
\hline benzo[a]fluorenone (baflo) & 0.00 & \pm & 0.00 & 0.00 & \pm & 0.00 & 0.00 & \pm & 0.00 & 0.00 & \pm & 0.00 & 0.00 & \pm & 0.00 & 0.01 & \pm & 0.00 \\
\hline benzo[b]fluorenone (bbflo) & 0.00 & \pm & 0.00 & 0.00 & \pm & 0.00 & 0.00 & \pm & 0.00 & 0.00 & \pm & 0.00 & 0.00 & \pm & 0.00 & 0.01 & \pm & 0.00 \\
\hline benzanthrenone (bao) & 0.00 & \pm & 0.00 & 0.00 & \pm & 0.00 & 0.00 & \pm & 0.00 & 0.00 & \pm & 0.00 & 0.00 & \pm & 0.00 & 0.01 & \pm & 0.00 \\
\hline phenanthrene (phe) & 0.00 & \pm & 0.00 & 0.00 & \pm & 0.00 & 0.00 & \pm & 0.00 & 0.00 & \pm & 0.00 & 0.00 & \pm & 0.00 & 0.03 & \pm & 0.01 \\
\hline anthracene (ant) & 0.00 & \pm & 0.00 & 0.00 & \pm & 0.00 & 0.00 & \pm & 0.00 & 0.00 & \pm & 0.00 & 0.00 & \pm & 0.00 & 0.01 & \pm & 0.00 \\
\hline fluoranthene (fla) & 0.00 & \pm & 0.00 & 0.00 & \pm & 0.00 & 0.00 & \pm & 0.00 & 0.01 & \pm & 0.00 & 0.01 & \pm & 0.00 & 0.05 & \pm & 0.01 \\
\hline pyrene (pyr) & 0.00 & \pm & 0.00 & 0.00 & \pm & 0.00 & 0.00 & \pm & 0.00 & 0.01 & \pm & 0.00 & 0.01 & \pm & 0.00 & 0.07 & \pm & 0.02 \\
\hline retene (ret) & 0.00 & \pm & 0.00 & 0.00 & \pm & 0.00 & 0.00 & \pm & 0.00 & 0.00 & \pm & 0.00 & 0.00 & \pm & 0.00 & 0.00 & \pm & 0.00 \\
\hline benz[a]anthracene (baa) & 0.00 & \pm & 0.00 & 0.00 & \pm & 0.00 & 0.00 & \pm & 0.00 & 0.00 & \pm & 0.00 & 0.00 & \pm & 0.00 & 0.02 & \pm & 0.01 \\
\hline $\begin{array}{l}\text { chrysene (chry) } \\
\text { benzo[b+ijfluoranthene }\end{array}$ & 0.00 & \pm & 0.00 & 0.00 & \pm & 0.00 & 0.00 & \pm & 0.00 & 0.00 & \pm & 0.00 & 0.00 & \pm & 0.00 & 0.04 & \pm & 0.02 \\
\hline (bbjfla) & 0.00 & \pm & 0.00 & 0.00 & \pm & 0.00 & 0.00 & \pm & 0.00 & 0.00 & \pm & 0.00 & 0.01 & \pm & 0.00 & 0.10 & \pm & 0.06 \\
\hline benzo[k]fluoranthene (bkfla) & 0.00 & \pm & 0.00 & 0.00 & \pm & 0.00 & 0.00 & \pm & 0.00 & 0.00 & \pm & 0.00 & 0.00 & \pm & 0.00 & 0.03 & \pm & 0.02 \\
\hline benzo[e]pyrene (bep) & 0.00 & \pm & 0.00 & 0.01 & \pm & 0.00 & 0.01 & \pm & 0.00 & 0.01 & \pm & 0.00 & 0.01 & \pm & 0.00 & 0.13 & \pm & 0.07 \\
\hline benzo[a]pyrene (bap) & 0.00 & \pm & 0.00 & 0.01 & \pm & 0.00 & 0.01 & \pm & 0.00 & 0.01 & \pm & 0.00 & 0.01 & \pm & 0.00 & 0.08 & \pm & 0.03 \\
\hline indeno[123cd]pyrene (ip) & 0.00 & \pm & 0.00 & 0.00 & \pm & 0.00 & 0.00 & \pm & 0.00 & 0.01 & \pm & 0.00 & 0.01 & \pm & 0.00 & 0.11 & \pm & 0.05 \\
\hline dibenz[ah]anthracene (dba) & 0.00 & \pm & 0.00 & 0.00 & \pm & 0.00 & 0.00 & \pm & 0.00 & 0.00 & \pm & 0.00 & 0.00 & \pm & 0.00 & 0.03 & \pm & 0.01 \\
\hline benzo[ghi]perylene (bgp) & 0.01 & \pm & 0.00 & 0.01 & \pm & 0.00 & 0.01 & \pm & 0.00 & 0.01 & \pm & 0.01 & 0.02 & \pm & 0.01 & 0.15 & \pm & 0.07 \\
\hline $\begin{array}{l}\text { coronene (cor) } \\
17 a(H) 21 B(H)-29 \text {-norhopane }\end{array}$ & 0.00 & \pm & 0.00 & 0.01 & \pm & 0.00 & 0.00 & \pm & 0.00 & 0.01 & \pm & 0.01 & 0.01 & \pm & 0.00 & 0.06 & \pm & 0.03 \\
\hline (norHop) & 0.03 & \pm & 0.00 & 0.03 & \pm & 0.01 & 0.03 & \pm & 0.01 & 0.02 & \pm & 0.00 & 0.05 & \pm & 0.04 & 0.30 & \pm & 0.06 \\
\hline $17 \mathrm{a}(\mathrm{H}) 21 \beta(\mathrm{H})$-hopane (Hop) & 0.03 & \pm & 0.01 & 0.03 & \pm & 0.01 & 0.02 & \pm & 0.01 & 0.02 & \pm & 0.00 & 0.04 & \pm & 0.03 & 0.27 & \pm & 0.07 \\
\hline $\mathrm{nC20}$ & 0.03 & \pm & 0.01 & 0.02 & \pm & 0.00 & 0.01 & \pm & 0.01 & 0.02 & \pm & 0.00 & 0.04 & \pm & 0.03 & 0.02 & \pm & 0.01 \\
\hline $\mathrm{nC21}$ & 0.09 & \pm & 0.05 & 0.07 & \pm & 0.06 & 0.04 & \pm & 0.04 & 0.05 & \pm & 0.00 & 0.08 & \pm & 0.05 & 0.13 & \pm & 0.03 \\
\hline $\mathrm{nC22}$ & 0.09 & \pm & 0.08 & 0.06 & \pm & 0.00 & 0.03 & \pm & 0.03 & 0.07 & \pm & 0.01 & 0.13 & \pm & 0.09 & 0.06 & \pm & 0.03 \\
\hline nC23 & 0.05 & \pm & 0.03 & 0.05 & \pm & 0.02 & 0.02 & \pm & 0.02 & 0.05 & \pm & 0.01 & 0.07 & \pm & 0.04 & 0.08 & \pm & 0.04 \\
\hline nC24 & 0.04 & \pm & 0.04 & 0.04 & \pm & 0.00 & 0.02 & \pm & 0.01 & 0.04 & \pm & 0.01 & 0.06 & \pm & 0.04 & 0.11 & \pm & 0.04 \\
\hline nC25 & 0.15 & \pm & 0.05 & 0.18 & \pm & 0.08 & 0.10 & \pm & 0.02 & 0.18 & \pm & 0.01 & 0.24 & \pm & 0.03 & 0.27 & \pm & 0.16 \\
\hline nC26 & 0.08 & \pm & 0.05 & 0.06 & \pm & 0.02 & 0.03 & \pm & 0.01 & 0.07 & \pm & 0.02 & 0.13 & \pm & 0.10 & 0.27 & \pm & 0.27 \\
\hline nC27 & 0.21 & \pm & 0.04 & 0.35 & \pm & 0.15 & 0.22 & \pm & 0.02 & 0.29 & \pm & 0.04 & 0.27 & \pm & 0.02 & 0.46 & \pm & 0.36 \\
\hline $\mathrm{nC} 28$ & | 0.08 & \pm & 0.05 & 0.08 & \pm & 0.01 & 0.04 & \pm & 0.01 & 0.08 & \pm & 0.03 & 0.06 & \pm & 0.01 & 0.16 & \pm & 0.16 \\
\hline
\end{tabular}




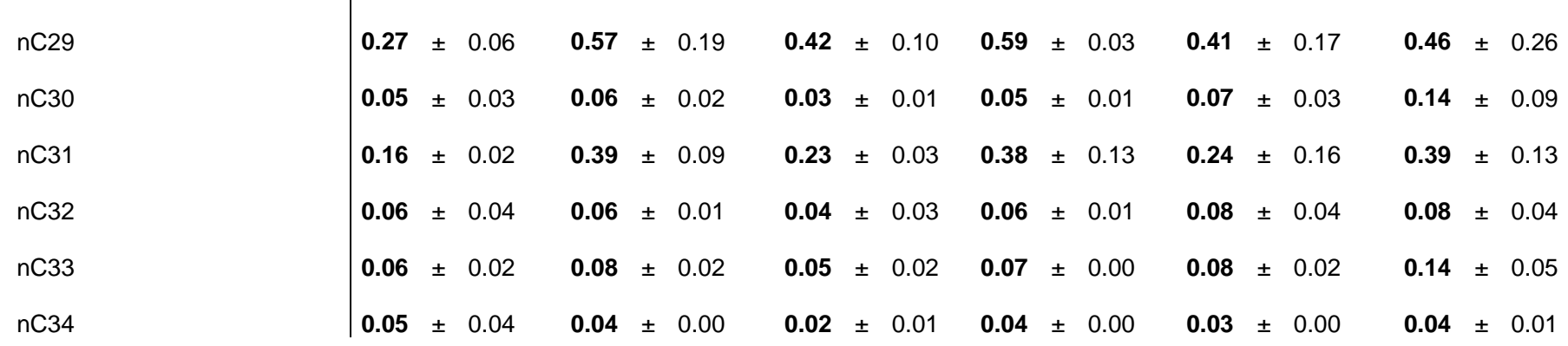

\section{Cold period}

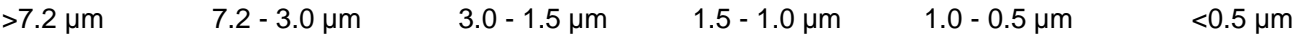

$\left(\mathrm{ng} / \mathrm{m}^{3}\right)$

succinic acid (SA)

glutaric acid (GA)

adipic acid (AdA)

pimelic acid (PA)

suberic acid (SbA)

azelaic acid (AzA)

glyceric acid (GyA)

malic acid (MA)

3-hydroxyglutaric acid (3HGA)

MBTCA

cis-pinonic acid (CPA)

pinic acid (PNA)

2-methylglyceric acid (2MGA)

C5-alkene triols (C5T)

2-methylthreitol (2MT1)

2-methylerythritol (2MT2)

phthalic acid $(\mathrm{PhA})$

terephthalic acid (TPhA)

nicotine (NIC)

galactosan (G)

mannosan (M)

levoglucosan (L)

xylitol (X)

mannitol (MaOL

$\alpha$-glucose ( $\alpha \mathrm{GL})$

$\beta$-glucose ( $\beta G L)$

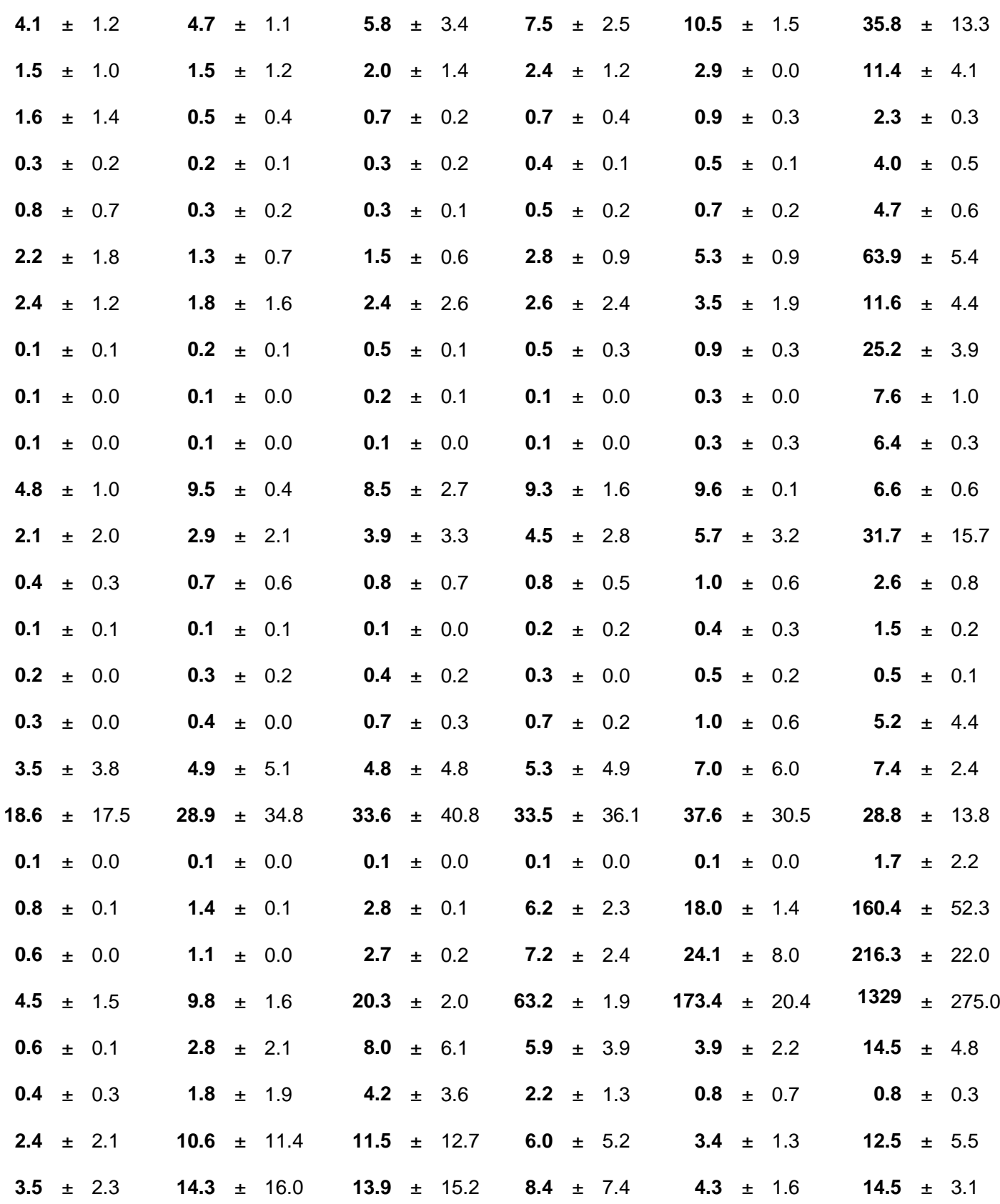




\begin{tabular}{|c|c|c|c|c|c|c|c|c|c|c|c|c|c|c|c|c|c|c|}
\hline dehydrabietic acid (DHA) & 1.2 & \pm & 0.8 & 4.4 & \pm & 1.1 & 6.8 & \pm & 1.7 & 24.2 & \pm & 7.3 & 81.2 & \pm & 5.7 & 350.0 & \pm & 94.1 \\
\hline sucrose (S) & 4.8 & \pm & 2.2 & 0.9 & \pm & 0.8 & 0.6 & \pm & 0.1 & 1.3 & \pm & 0.2 & 1.1 & \pm & 0.2 & 10.8 & \pm & 2.8 \\
\hline mycose (My) & 0.4 & \pm & 0.2 & 2.8 & \pm & 3.7 & 2.7 & \pm & 2.6 & 1.9 & \pm & 1.2 & 0.9 & \pm & 0.5 & 1.4 & \pm & 0.4 \\
\hline C16:0 & 14.6 & \pm & 13.7 & 8.4 & \pm & 1.8 & 5.2 & \pm & 2.0 & 7.5 & \pm & 2.7 & 13.1 & \pm & 2.2 & 73.7 & \pm & 3.2 \\
\hline $\mathrm{C} 17: 0$ & 0.7 & \pm & 0.6 & 0.4 & \pm & 0.0 & 0.3 & \pm & 0.0 & 0.6 & \pm & 0.1 & 0.9 & \pm & 0.0 & 4.4 & \pm & 0.2 \\
\hline C18:1 & 9.1 & \pm & 12.1 & 1.6 & \pm & 0.7 & 1.4 & \pm & 1.0 & 1.2 & \pm & 0.6 & 3.8 & \pm & 1.6 & 17.4 & \pm & 0.8 \\
\hline C18:0 & 3.3 & \pm & 3.2 & 4.0 & \pm & 0.7 & 2.3 & \pm & 0.6 & 2.9 & \pm & 0.8 & 5.2 & \pm & 0.6 & 49.3 & \pm & 2.1 \\
\hline C19:0 & 0.2 & \pm & 0.1 & 0.2 & \pm & 0.1 & 0.1 & \pm & 0.0 & 0.4 & \pm & 0.0 & 1.1 & \pm & 0.1 & 3.4 & \pm & 0.1 \\
\hline $\mathrm{C} 20: 0$ & 0.4 & \pm & 0.4 & 0.4 & \pm & 0.1 & 0.3 & \pm & 0.0 & 0.8 & \pm & 0.2 & 2.2 & \pm & 0.6 & 12.0 & \pm & 0.5 \\
\hline $\mathrm{C} 21: 0$ & 0.1 & \pm & 0.0 & 0.1 & \pm & 0.0 & 0.1 & \pm & 0.0 & 0.3 & \pm & 0.1 & 0.8 & \pm & 0.3 & 5.0 & \pm & 0.2 \\
\hline fluorenone (flo) & 0.02 & \pm & 0.01 & 0.02 & \pm & 0.01 & 0.01 & \pm & 0.01 & 0.01 & \pm & 0.00 & 0.02 & \pm & 0.00 & 0.14 & \pm & 0.09 \\
\hline phenanthraquinone (pheno) & 0.10 & \pm & 0.12 & 0.12 & \pm & 0.12 & 0.08 & \pm & 0.08 & 0.07 & \pm & 0.04 & 0.10 & \pm & 0.03 & 0.81 & \pm & 0.47 \\
\hline anthracenequinone (anto) & 0.03 & \pm & 0.03 & 0.04 & \pm & 0.04 & 0.03 & \pm & 0.02 & 0.03 & \pm & 0.02 & 0.03 & \pm & 0.02 & 0.22 & \pm & 0.11 \\
\hline benzo[a]fluorenone (baflo) & 0.02 & \pm & 0.03 & 0.04 & \pm & 0.04 & 0.02 & \pm & 0.01 & 0.01 & \pm & 0.01 & 0.03 & \pm & 0.02 & 0.59 & \pm & 0.31 \\
\hline benzo[b]fluorenone (bbflo) & 0.02 & \pm & 0.03 & 0.04 & \pm & 0.05 & 0.01 & \pm & 0.01 & 0.01 & \pm & 0.01 & 0.03 & \pm & 0.03 & 0.79 & \pm & 0.25 \\
\hline benzanthrenone (bao) & 0.04 & \pm & 0.05 & 0.08 & \pm & 0.10 & 0.03 & \pm & 0.01 & 0.03 & \pm & 0.03 & 0.07 & \pm & 0.07 & 1.15 & \pm & 0.17 \\
\hline phenanthrene (phe) & 0.02 & \pm & 0.02 & 0.03 & \pm & 0.03 & 0.02 & \pm & 0.01 & 0.01 & \pm & 0.01 & 0.02 & \pm & 0.01 & 0.26 & \pm & 0.12 \\
\hline anthracene (ant) & 0.00 & \pm & 0.00 & 0.00 & \pm & 0.00 & 0.00 & \pm & 0.00 & 0.00 & \pm & 0.00 & 0.00 & \pm & 0.00 & 0.05 & \pm & 0.01 \\
\hline fluoranthene (fla) & 0.08 & \pm & 0.08 & 0.14 & \pm & 0.13 & 0.06 & \pm & 0.03 & 0.05 & \pm & 0.02 & 0.08 & \pm & 0.03 & 1.23 & \pm & 0.73 \\
\hline pyrene (pyr) & 0.09 & \pm & 0.08 & 0.17 & \pm & 0.16 & 0.07 & \pm & 0.03 & 0.06 & \pm & 0.02 & 0.10 & \pm & 0.05 & 1.63 & \pm & 0.92 \\
\hline retene (ret) & 0.25 & \pm & 0.30 & 0.43 & \pm & 0.48 & 0.19 & \pm & 0.17 & 0.12 & \pm & 0.00 & 0.22 & \pm & 0.01 & 2.03 & \pm & 1.49 \\
\hline benz[a]anthracene (baa) & 0.05 & \pm & 0.05 & 0.15 & \pm & 0.18 & 0.03 & \pm & 0.01 & 0.03 & \pm & 0.03 & 0.10 & \pm & 0.11 & 1.43 & \pm & 0.71 \\
\hline $\begin{array}{l}\text { chrysene (chry) } \\
\text { benzo[b+i]fluoranthene }\end{array}$ & 0.07 & \pm & 0.08 & 0.18 & \pm & 0.21 & 0.05 & \pm & 0.02 & 0.05 & \pm & 0.04 & 0.12 & \pm & 0.12 & 1.55 & \pm & 0.82 \\
\hline (bbjfla) & 0.09 & \pm & 0.10 & 0.24 & \pm & 0.29 & 0.05 & \pm & 0.01 & 0.08 & \pm & 0.09 & 0.22 & \pm & 0.26 & 2.02 & \pm & 0.47 \\
\hline benzo[k]fluoranthene (bkfla) & 0.02 & \pm & 0.03 & 0.07 & \pm & 0.09 & 0.01 & \pm & 0.00 & 0.02 & \pm & 0.03 & 0.06 & \pm & 0.07 & 0.73 & \pm & 0.20 \\
\hline benzo[e]pyrene (bep) & 0.07 & \pm & 0.07 & 0.15 & \pm & 0.16 & 0.05 & \pm & 0.01 & 0.06 & \pm & 0.06 & 0.13 & \pm & 0.13 & 1.04 & \pm & 0.27 \\
\hline benzo[a]pyrene (bap) & 0.07 & \pm & 0.07 & 0.17 & \pm & 0.21 & 0.04 & \pm & 0.00 & 0.06 & \pm & 0.07 & 0.16 & \pm & 0.20 & 0.99 & \pm & 0.27 \\
\hline indeno[123cd]pyrene (ip) & 0.06 & \pm & 0.06 & 0.14 & \pm & 0.16 & 0.04 & \pm & 0.00 & 0.06 & \pm & 0.07 & 0.14 & \pm & 0.16 & 1.14 & \pm & 0.29 \\
\hline dibenz[ah]anthracene (dba) & 0.02 & \pm & 0.02 & 0.04 & \pm & 0.04 & 0.01 & \pm & 0.00 & 0.01 & \pm & 0.02 & 0.03 & \pm & 0.04 & 0.35 & \pm & 0.09 \\
\hline benzo[ghi]perylene (bgp) & 0.06 & \pm & 0.06 & 0.13 & \pm & 0.13 & 0.05 & \pm & 0.00 & 0.06 & \pm & 0.06 & 0.13 & \pm & 0.14 & 0.82 & \pm & 0.19 \\
\hline $\begin{array}{l}\text { coronene (cor) } \\
17 a(H) 21 B(H)-29 \text {-norhopane }\end{array}$ & 0.03 & \pm & 0.02 & 0.06 & \pm & 0.06 & 0.02 & \pm & 0.00 & 0.03 & \pm & 0.03 & 0.07 & \pm & 0.07 & 0.44 & \pm & 0.09 \\
\hline (norHop) & 0.04 & \pm & 0.02 & 0.05 & \pm & 0.01 & 0.03 & \pm & 0.01 & 0.02 & \pm & 0.01 & 0.04 & \pm & 0.00 & 0.12 & \pm & 0.00 \\
\hline $17 a(H) 21 \beta(H)$-hopane (Hop) & 0.03 & \pm & 0.02 & 0.03 & \pm & 0.01 & 0.02 & \pm & 0.00 & 0.02 & \pm & 0.00 & 0.03 & \pm & 0.01 & 0.13 & \pm & 0.01 \\
\hline $\mathrm{nC20}$ & 0.10 & \pm & 0.04 & 0.11 & \pm & 0.10 & 0.07 & \pm & 0.04 & 0.04 & \pm & 0.01 & 0.07 & \pm & 0.01 & 0.65 & \pm & 0.42 \\
\hline $\mathrm{nC} 21$ & 0.18 & \pm & 0.00 & 0.24 & \pm & 0.09 & 0.19 & \pm & 0.10 & 0.12 & \pm & 0.10 & 0.13 & \pm & 0.04 & 1.40 & \pm & 1.07 \\
\hline nC22 & 0.17 & \pm & 0.11 & 0.28 & \pm & 0.30 & 0.09 & \pm & 0.05 & 0.07 & \pm & 0.03 & 0.12 & \pm & 0.04 & 2.16 & \pm & 1.60 \\
\hline $\mathrm{nC23}$ & 0.24 & \pm & 0.10 & 0.38 & \pm & 0.39 & 0.11 & \pm & 0.02 & 0.09 & \pm & 0.07 & 0.16 & \pm & 0.11 & 2.71 & \pm & 1.27 \\
\hline
\end{tabular}




\begin{tabular}{|c|c|c|c|c|c|c|c|c|c|c|c|c|c|c|c|c|c|c|}
\hline nC24 & 0.22 & \pm & 0.16 & 0.38 & \pm & 0.43 & 0.09 & \pm & 0.03 & 0.08 & \pm & 0.07 & 0.16 & \pm & 0.13 & 2.05 & \pm & 0.75 \\
\hline nC25 & 0.29 & \pm & 0.15 & 0.45 & \pm & 0.47 & 0.12 & \pm & 0.04 & 0.12 & \pm & 0.09 & 0.20 & \pm & 0.15 & 1.74 & \pm & 0.33 \\
\hline nC26 & 0.25 & \pm & 0.18 & 0.42 & \pm & 0.49 & 0.11 & \pm & 0.04 & 0.11 & \pm & 0.08 & 0.20 & \pm & 0.18 & 1.12 & \pm & 0.18 \\
\hline nC27 & 0.31 & \pm & 0.13 & 0.50 & \pm & 0.35 & 0.19 & \pm & 0.01 & 0.17 & \pm & 0.15 & 0.27 & \pm & 0.19 & 1.39 & \pm & 0.11 \\
\hline nC28 & 0.24 & \pm & 0.16 & 0.38 & \pm & 0.42 & 0.11 & \pm & 0.04 & 0.12 & \pm & 0.09 & 0.19 & \pm & 0.17 & 0.96 & \pm & 0.02 \\
\hline nC29 & 0.43 & \pm & 0.21 & 0.64 & \pm & 0.33 & 0.35 & \pm & 0.01 & 0.29 & \pm & 0.24 & 0.52 & \pm & 0.28 & 2.48 & \pm & 1.06 \\
\hline nC30 & 0.17 & \pm & 0.15 & 0.28 & \pm & 0.29 & 0.09 & \pm & 0.04 & 0.07 & \pm & 0.05 & 0.16 & \pm & 0.13 & 0.60 & \pm & 0.16 \\
\hline nC31 & 0.30 & \pm & 0.21 & 0.49 & \pm & 0.28 & 0.25 & \pm & 0.04 & 0.22 & \pm & 0.20 & 0.48 & \pm & 0.34 & 2.43 & \pm & 1.80 \\
\hline nC32 & 0.17 & \pm & 0.09 & 0.22 & \pm & 0.22 & 0.07 & \pm & 0.04 & 0.06 & \pm & 0.01 & 0.12 & \pm & 0.09 & 0.44 & \pm & 0.05 \\
\hline nC33 & 0.17 & \pm & 0.06 & 0.22 & \pm & 0.21 & 0.08 & \pm & 0.04 & 0.04 & \pm & 0.01 & 0.13 & \pm & 0.06 & 0.60 & \pm & 0.08 \\
\hline nC34 & 0.14 & \pm & 0.05 & 0.16 & \pm & 0.16 & 0.06 & \pm & 0.02 & 0.04 & \pm & 0.00 & 0.06 & \pm & 0.03 & 0.19 & \pm & 0.09 \\
\hline
\end{tabular}

Table S2c-d. Summary of organic tracer compound concentrations in Warm and Cold sampling periods in the Urban site.

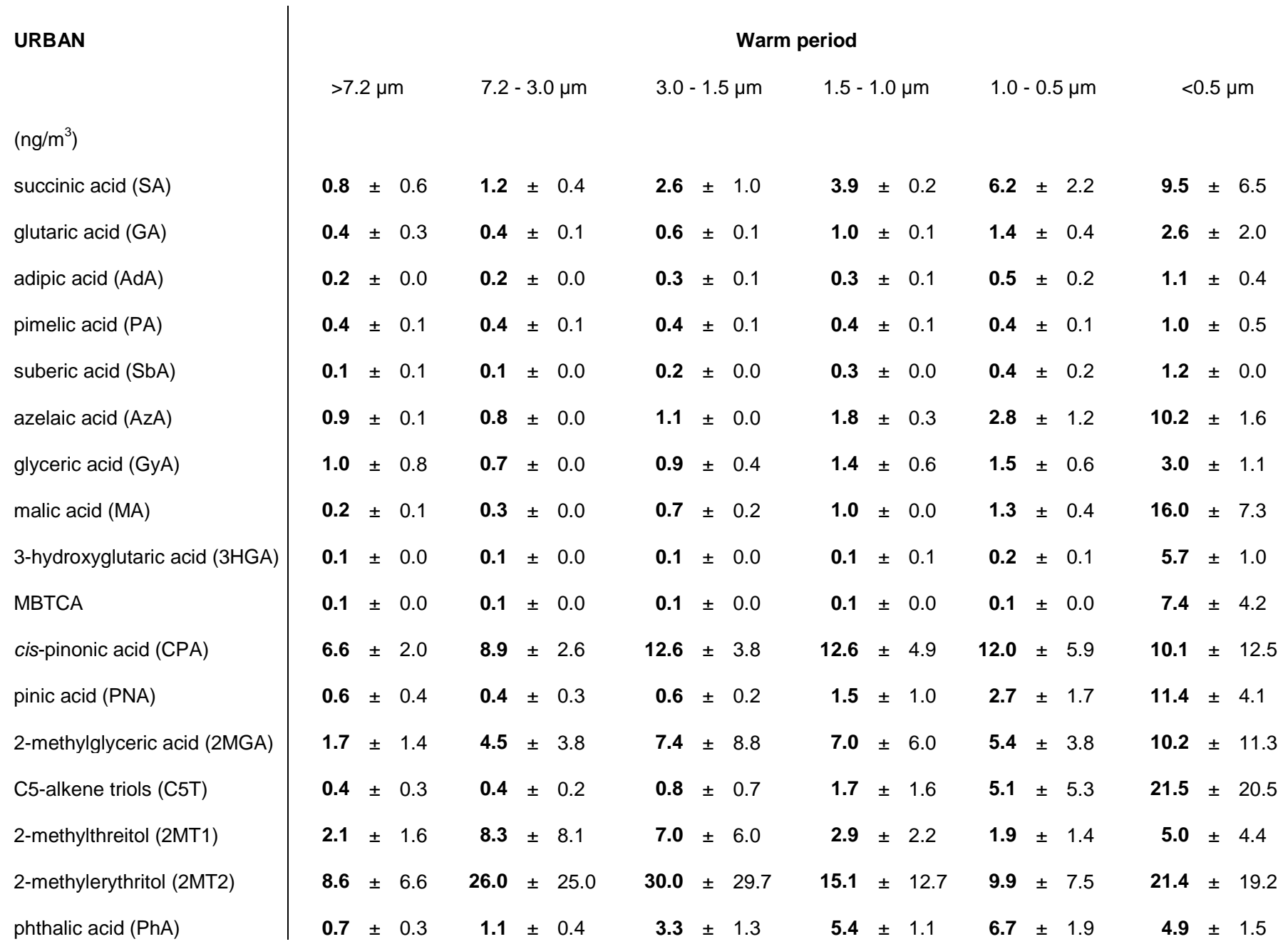




\begin{tabular}{|c|c|c|c|c|c|c|c|c|c|c|c|c|c|c|c|c|c|c|}
\hline terephthalic acid (TPhA) & 8.7 & \pm & 4.4 & 4.9 & \pm & 2.0 & 8.0 & \pm & 2.1 & 25.6 & \pm & 10.6 & 38.4 & \pm & 11.7 & 39.5 & \pm & 14.1 \\
\hline nicotine (NIC) & 0.2 & \pm & 0.1 & 0.3 & \pm & 0.1 & 0.4 & \pm & 0.2 & 0.6 & \pm & 0.5 & 1.6 & \pm & 1.7 & 12.7 & \pm & 7.2 \\
\hline galactosan $(\mathrm{G})$ & 0.1 & \pm & 0.0 & 0.2 & \pm & 0.1 & 0.3 & \pm & 0.2 & 0.2 & \pm & 0.2 & 0.3 & \pm & 0.3 & 0.3 & \pm & 0.4 \\
\hline mannosan (M) & 0.0 & \pm & 0.0 & 0.1 & \pm & 0.1 & 0.2 & \pm & 0.2 & 0.2 & \pm & 0.3 & 0.4 & \pm & 0.4 & 0.6 & \pm & 0.8 \\
\hline levoglucosan (L) & 0.4 & \pm & 0.1 & 0.8 & \pm & 0.4 & 1.5 & \pm & 1.1 & 2.4 & \pm & 2.5 & 4.4 & \pm & 6.0 & 8.2 & \pm & 10.9 \\
\hline xylitol (X) & 20.3 & \pm & 10.0 & 23.3 & \pm & 5.2 & 16.1 & \pm & 4.6 & 4.1 & \pm & 0.6 & 1.5 & \pm & 0.2 & 5.7 & \pm & 3.2 \\
\hline mannitol (MaOL & 6.6 & \pm & 1.5 & 10.5 & \pm & 3.3 & 6.9 & \pm & 2.3 & 1.6 & \pm & 0.4 & 0.5 & \pm & 0.0 & 2.0 & \pm & 0.4 \\
\hline$\alpha$-glucose ( $\alpha \mathrm{GL})$ & 35.4 & \pm & 17.4 & 32.1 & \pm & 1.7 & 18.1 & \pm & 4.0 & 6.1 & \pm & 1.1 & 2.3 & \pm & 0.1 & 11.6 & \pm & 8.0 \\
\hline$\beta$-glucose ( $\beta G L)$ & 35.1 & \pm & 11.4 & 30.6 & \pm & 2.3 & 18.9 & \pm & 4.0 & 6.6 & \pm & 1.4 & 2.5 & \pm & 0.1 & 12.6 & \pm & 8.1 \\
\hline dehydrabietic acid (DHA) & 1.9 & \pm & 1.0 & 1.1 & \pm & 0.4 & 0.4 & \pm & 0.1 & 0.3 & \pm & 0.0 & 0.3 & \pm & 0.1 & 1.3 & \pm & 0.9 \\
\hline sucrose (S) & 180.7 & \pm & 58.7 & 102.5 & \pm & 28.2 & 51.7 & \pm & 21.6 & 10.9 & \pm & 3.1 & 2.9 & \pm & 1.1 & 63.1 & \pm & 37.0 \\
\hline mycose (My) & 35.3 & \pm & 22.1 & 34.2 & \pm & 13.1 & 14.3 & \pm & 5.4 & 2.6 & \pm & 1.0 & 0.6 & \pm & 0.3 & 4.0 & \pm & 2.0 \\
\hline C16:0 & 22.5 & \pm & 5.4 & 23.7 & \pm & 6.0 & 16.5 & \pm & 3.7 & 16.5 & \pm & 2.5 & 19.8 & \pm & 5.6 & 37.4 & \pm & 10.8 \\
\hline C17:0 & 1.6 & \pm & 0.9 & 0.8 & \pm & 0.2 & 0.7 & \pm & 0.2 & 0.8 & \pm & 0.2 & 0.8 & \pm & 0.3 & 1.8 & \pm & 0.4 \\
\hline C18:1 & 8.8 & \pm & 6.0 & 3.5 & \pm & 1.3 & 3.0 & \pm & 1.3 & 2.8 & \pm & 0.9 & 5.6 & \pm & 4.4 & 2.8 & \pm & 2.0 \\
\hline C18:0 & 16.6 & \pm & 4.1 & 14.4 & \pm & 5.4 & 9.4 & \pm & 3.7 & 8.8 & \pm & 3.5 & 15.0 & \pm & 11.2 & 14.9 & \pm & 4.2 \\
\hline C19:0 & 3.8 & \pm & 3.4 & 1.4 & \pm & 1.7 & 0.2 & \pm & 0.1 & 0.2 & \pm & 0.1 & 0.3 & \pm & 0.1 & 0.9 & \pm & 0.2 \\
\hline C20:0 & 2.9 & \pm & 0.8 & 2.2 & \pm & 0.7 & 1.2 & \pm & 0.4 & 0.9 & \pm & 0.3 & 1.3 & \pm & 0.8 & 2.5 & \pm & 0.2 \\
\hline C21:0 & 0.2 & \pm & 0.0 & 0.2 & \pm & 0.0 & 0.2 & \pm & 0.1 & 0.2 & \pm & 0.1 & 0.2 & \pm & 0.1 & 0.9 & \pm & 0.1 \\
\hline fluorenone (flo) & 0.00 & \pm & 0.00 & 0.01 & \pm & 0.00 & 0.00 & \pm & 0.00 & 0.00 & \pm & 0.00 & 0.00 & \pm & 0.00 & 0.04 & \pm & 0.02 \\
\hline phenanthraquinone (pheno) & 0.00 & \pm & 0.00 & 0.00 & \pm & 0.00 & 0.00 & \pm & 0.00 & 0.00 & \pm & 0.00 & 0.00 & \pm & 0.00 & 0.03 & \pm & 0.00 \\
\hline anthracenequinone (anto) & 0.01 & \pm & 0.00 & 0.01 & \pm & 0.00 & 0.00 & \pm & 0.00 & 0.01 & \pm & 0.00 & 0.01 & \pm & 0.00 & 0.06 & \pm & 0.01 \\
\hline benzo[a]fluorenone (baflo) & 0.00 & \pm & 0.00 & 0.01 & \pm & 0.00 & 0.00 & \pm & 0.00 & 0.01 & \pm & 0.00 & 0.01 & \pm & 0.00 & 0.07 & \pm & 0.01 \\
\hline benzo[b]fluorenone (bbflo) & 0.00 & \pm & 0.00 & 0.00 & \pm & 0.00 & 0.00 & \pm & 0.00 & 0.00 & \pm & 0.00 & 0.00 & \pm & 0.00 & 0.05 & \pm & 0.01 \\
\hline benzanthrenone (bao) & 0.00 & \pm & 0.00 & 0.00 & \pm & 0.00 & 0.00 & \pm & 0.00 & 0.00 & \pm & 0.00 & 0.01 & \pm & 0.00 & 0.06 & \pm & 0.00 \\
\hline phenanthrene (phe) & 0.01 & \pm & 0.01 & 0.02 & \pm & 0.01 & 0.01 & \pm & 0.01 & 0.01 & \pm & 0.01 & 0.02 & \pm & 0.02 & 0.12 & \pm & 0.04 \\
\hline anthracene (ant) & 0.00 & \pm & 0.00 & 0.00 & \pm & 0.00 & 0.00 & \pm & 0.00 & 0.00 & \pm & 0.00 & 0.00 & \pm & 0.00 & 0.02 & \pm & 0.00 \\
\hline fluoranthene (fla) & 0.02 & \pm & 0.01 & 0.03 & \pm & 0.01 & 0.02 & \pm & 0.01 & 0.02 & \pm & 0.01 & 0.03 & \pm & 0.02 & 0.19 & \pm & 0.04 \\
\hline pyrene (pyr) & 0.05 & \pm & 0.02 & 0.06 & \pm & 0.04 & 0.03 & \pm & 0.02 & 0.03 & \pm & 0.02 & 0.05 & \pm & 0.04 & 0.33 & \pm & 0.08 \\
\hline retene (ret) & 0.00 & \pm & 0.00 & 0.00 & \pm & 0.00 & 0.00 & \pm & 0.00 & 0.00 & \pm & 0.00 & 0.00 & \pm & 0.00 & 0.01 & \pm & 0.00 \\
\hline benz[a]anthracene (baa) & 0.01 & \pm & 0.00 & 0.01 & \pm & 0.00 & 0.01 & \pm & 0.00 & 0.01 & \pm & 0.01 & 0.01 & \pm & 0.01 & 0.07 & \pm & 0.01 \\
\hline $\begin{array}{l}\text { chrysene (chry) } \\
\text { benzo[b+i]fluoranthene }\end{array}$ & 0.02 & \pm & 0.01 & 0.02 & \pm & 0.01 & 0.01 & \pm & 0.01 & 0.02 & \pm & 0.01 & 0.03 & \pm & 0.02 & 0.12 & \pm & 0.02 \\
\hline (bbjfla) & 0.02 & \pm & 0.01 & 0.02 & \pm & 0.01 & 0.01 & \pm & 0.01 & 0.02 & \pm & 0.01 & 0.04 & \pm & 0.03 & 0.14 & \pm & 0.02 \\
\hline benzo[k]fluoranthene (bkfla) & 0.00 & \pm & 0.00 & 0.00 & \pm & 0.00 & 0.00 & \pm & 0.00 & 0.00 & \pm & 0.00 & 0.01 & \pm & 0.00 & 0.04 & \pm & 0.01 \\
\hline benzo[e]pyrene (bep) & 0.04 & \pm & 0.03 & 0.05 & \pm & 0.02 & 0.03 & \pm & 0.02 & 0.03 & \pm & 0.02 & 0.05 & \pm & 0.04 & 0.17 & \pm & 0.02 \\
\hline benzo[a]pyrene (bap) & 0.03 & \pm & 0.02 & 0.03 & \pm & 0.01 & 0.02 & \pm & 0.01 & 0.02 & \pm & 0.01 & 0.03 & \pm & 0.02 & 0.09 & \pm & 0.02 \\
\hline indeno[123cd]pyrene (ip) & 0.02 & \pm & 0.01 & 0.02 & \pm & 0.01 & 0.01 & \pm & 0.01 & 0.02 & \pm & 0.01 & 0.03 & \pm & 0.02 & 0.12 & \pm & 0.03 \\
\hline
\end{tabular}




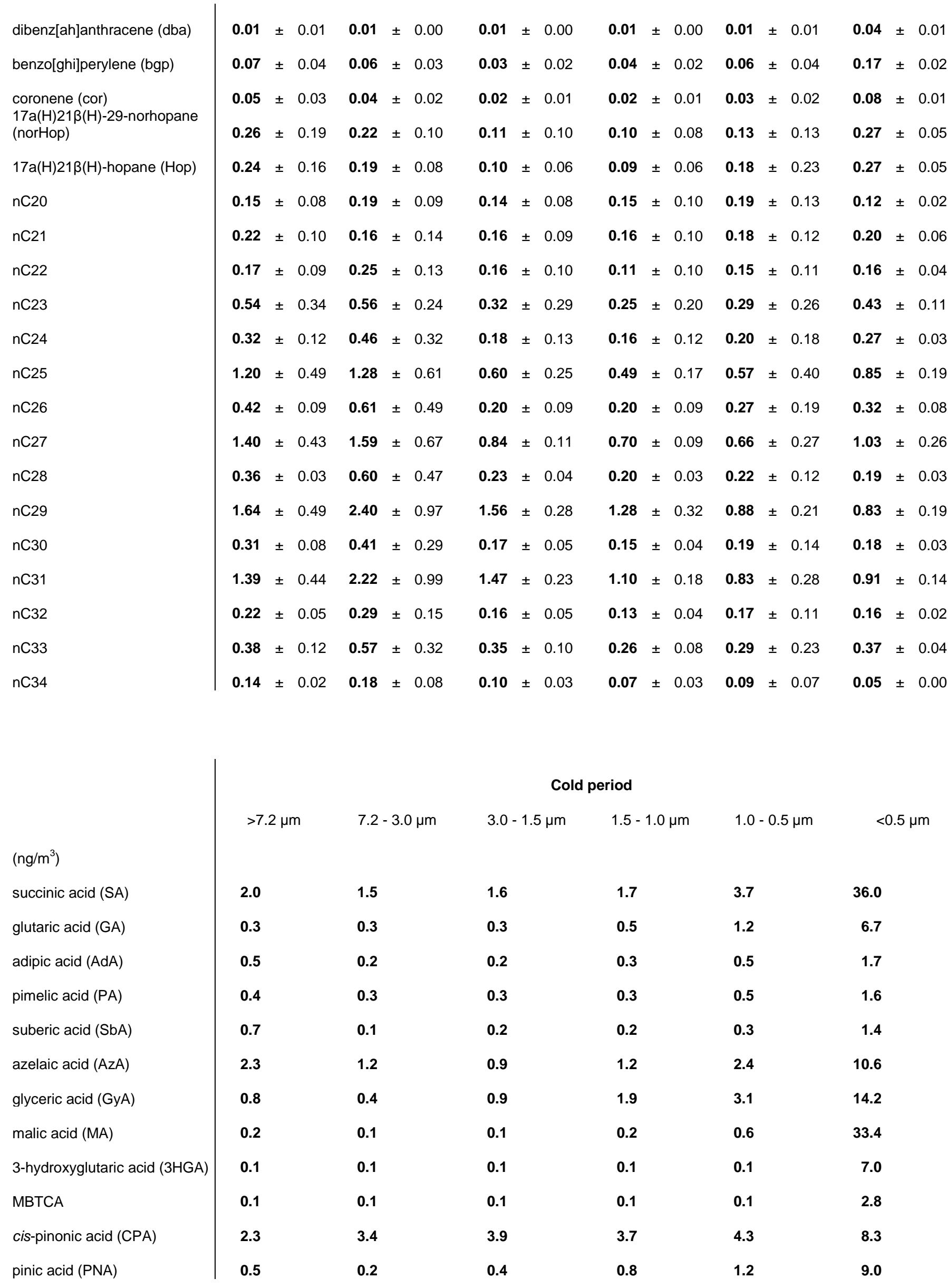




\begin{tabular}{|c|c|c|c|c|c|c|}
\hline 2-methylglyceric acid (2MGA) & 1.5 & 0.7 & 0.8 & 1.1 & 1.5 & 2.9 \\
\hline C5-alkene triols (C5T) & 1.0 & 0.1 & 0.1 & 0.2 & 0.2 & 1.2 \\
\hline 2-methylthreitol (2MT1) & 0.3 & 0.1 & 0.1 & 0.3 & 0.3 & 0.8 \\
\hline 2-methylerythritol (2MT2) & 1.0 & 0.4 & 0.6 & 0.7 & 1.4 & 9.7 \\
\hline phthalic acid (PhA) & 0.6 & 0.7 & 0.8 & 1.4 & 3.5 & 11.5 \\
\hline terephthalic acid (TPhA) & 26.2 & 10.7 & 20.5 & 38.9 & 53.0 & 21.4 \\
\hline nicotine (NIC) & 0.1 & 0.1 & 0.1 & 0.4 & 0.6 & 14.6 \\
\hline galactosan $(\mathrm{G})$ & 0.2 & 0.4 & 0.9 & 1.3 & 2.9 & 9.5 \\
\hline mannosan (M) & 0.1 & 0.2 & 0.4 & 0.7 & 1.8 & 10.1 \\
\hline levoglucosan (L) & 1.4 & 2.5 & 4.5 & 9.9 & 27.0 & 110.2 \\
\hline xylitol (X) & 3.0 & 4.8 & 4.2 & 1.6 & 1.1 & 3.2 \\
\hline mannitol (MaOL & 0.7 & 0.7 & 0.5 & 0.3 & 0.2 & 0.9 \\
\hline$\alpha$-glucose ( $\alpha \mathrm{GL})$ & 14.8 & 14.5 & 8.4 & 4.2 & 1.8 & 5.3 \\
\hline$\beta$-glucose ( $\beta G L)$ & 17.7 & 16.5 & 10.9 & 4.9 & 2.2 & 5.6 \\
\hline dehydrabietic acid (DHA) & 3.2 & 2.7 & 1.1 & 0.9 & 1.8 & 13.5 \\
\hline sucrose (S) & 52.0 & 60.1 & 45.9 & 10.0 & 6.0 & 16.1 \\
\hline mycose (My) & 1.4 & 1.9 & 1.3 & 0.4 & 0.2 & 0.4 \\
\hline C16:0 & 40.3 & 23.5 & 13.3 & 10.1 & 12.2 & 44.1 \\
\hline C17:0 & 1.6 & 1.0 & 0.6 & 0.5 & 0.6 & 2.1 \\
\hline C18:1 & 7.8 & 4.2 & 3.5 & 0.9 & 1.1 & 13.1 \\
\hline C18:0 & 26.8 & 20.9 & 12.1 & 4.7 & 5.0 & 34.1 \\
\hline C19:0 & 0.1 & 0.3 & 0.1 & 0.1 & 0.2 & 0.9 \\
\hline $\mathrm{C} 20: 0$ & 1.7 & 1.1 & 0.6 & 0.4 & 0.6 & 4.1 \\
\hline $\mathrm{C} 21: 0$ & 0.2 & 0.1 & 0.1 & 0.1 & 0.2 & 1.5 \\
\hline fluorenone (flo) & 0.01 & 0.01 & 0.01 & 0.01 & 0.01 & 0.07 \\
\hline phenanthraquinone (pheno) & 0.00 & 0.01 & 0.01 & 0.01 & 0.01 & 0.10 \\
\hline anthracenequinone (anto) & 0.01 & 0.01 & 0.01 & 0.01 & 0.01 & 0.11 \\
\hline benzo[a]fluorenone (baflo) & 0.01 & 0.01 & 0.01 & 0.01 & 0.02 & 0.21 \\
\hline benzo[b]fluorenone (bbflo) & 0.00 & 0.01 & 0.01 & 0.01 & 0.01 & 0.18 \\
\hline benzanthrenone (bao) & 0.00 & 0.01 & 0.01 & 0.01 & 0.02 & 0.18 \\
\hline phenanthrene (phe) & 0.02 & 0.02 & 0.01 & 0.01 & 0.02 & 0.16 \\
\hline anthracene (ant) & 0.00 & 0.00 & 0.00 & 0.00 & 0.00 & 0.04 \\
\hline fluoranthene (fla) & 0.03 & 0.03 & 0.03 & 0.03 & 0.04 & 0.36 \\
\hline pyrene (pyr) & 0.05 & 0.06 & 0.05 & 0.04 & 0.06 & 0.62 \\
\hline retene (ret) & 0.01 & 0.01 & 0.01 & 0.01 & 0.01 & 0.08 \\
\hline benz[a]anthracene (baa) & 0.01 & 0.01 & 0.01 & 0.01 & 0.02 & 0.27 \\
\hline chrysene (chry) & 0.01 & 0.02 & 0.02 & 0.03 & 0.05 & 0.44 \\
\hline
\end{tabular}




\begin{tabular}{|c|c|c|c|c|c|c|}
\hline $\begin{array}{l}\text { benzo[b+j]fluoranthene } \\
\text { (bbjfla) }\end{array}$ & 0.01 & 0.02 & 0.02 & 0.03 & 0.07 & 0.48 \\
\hline benzo[k]fluoranthene (bkfla) & 0.00 & 0.00 & 0.00 & 0.01 & 0.02 & 0.15 \\
\hline benzo[e]pyrene (bep) & 0.03 & 0.04 & 0.03 & 0.04 & 0.08 & 0.43 \\
\hline benzo[a]pyrene (bap) & 0.02 & 0.02 & 0.02 & 0.03 & 0.06 & 0.33 \\
\hline indeno[123cd]pyrene (ip) & 0.02 & 0.02 & 0.02 & 0.02 & 0.05 & 0.40 \\
\hline dibenz[ah]anthracene (dba) & 0.01 & 0.01 & 0.01 & 0.01 & 0.02 & 0.11 \\
\hline benzo[ghi]perylene (bgp) & 0.05 & 0.05 & 0.04 & 0.04 & 0.08 & 0.43 \\
\hline $\begin{array}{l}\text { coronene (cor) } \\
17 \mathrm{a}(\mathrm{H}) 21 \beta(\mathrm{H})-29 \text {-norhopane }\end{array}$ & 0.04 & 0.03 & 0.02 & 0.02 & 0.04 & 0.22 \\
\hline (norHop) & 0.22 & 0.20 & 0.11 & 0.08 & 0.08 & 0.40 \\
\hline $17 \mathrm{a}(\mathrm{H}) 21 \beta(\mathrm{H})$-hopane (Hop) & 0.21 & 0.21 & 0.11 & 0.07 & 0.08 & 0.41 \\
\hline $\mathrm{nC20}$ & 0.14 & 0.14 & 0.10 & 0.09 & 0.08 & 0.32 \\
\hline $\mathrm{nC21}$ & 0.17 & 0.22 & 0.14 & 0.14 & 0.11 & 0.45 \\
\hline nC22 & 0.18 & 0.19 & 0.11 & 0.09 & 0.12 & 0.63 \\
\hline nC23 & 0.28 & 0.30 & 0.17 & 0.16 & 0.20 & 1.13 \\
\hline nC24 & 0.22 & 0.25 & 0.15 & 0.16 & 0.22 & 1.10 \\
\hline nC25 & 0.35 & 0.40 & 0.23 & 0.26 & 0.35 & 1.55 \\
\hline nC26 & 0.26 & 0.28 & 0.16 & 0.16 & 0.22 & 0.66 \\
\hline nC27 & 0.50 & 0.59 & 0.35 & 0.36 & 0.48 & 1.46 \\
\hline nC28 & 0.29 & 0.36 & 0.19 & 0.14 & 0.15 & 0.26 \\
\hline nC29 & 0.51 & 0.70 & 0.56 & 0.38 & 0.45 & 0.91 \\
\hline nC30 & 0.31 & 0.32 & 0.19 & 0.12 & 0.11 & 0.24 \\
\hline nC31 & 0.53 & 0.67 & 0.52 & 0.33 & 0.37 & 1.11 \\
\hline nC32 & 0.32 & 0.32 & 0.19 & 0.11 & 0.09 & 0.21 \\
\hline nC33 & 0.29 & 0.28 & 0.22 & 0.15 & 0.13 & 0.46 \\
\hline nC34 & 0.18 & 0.16 & 0.13 & 0.07 & 0.05 & 0.09 \\
\hline
\end{tabular}


Figure S1. Two potential Biomass Burning Organic Aerosol component loadings (1) and scores (2) from separate MCR-ALS analysis of the data from the Rural site. R_COLD_1.x are the fall samples, while and R_COLD_2.x are the winter samples. The x.6 samples are the $P M<0.5 \mu m$ fraction of the samples.

1)

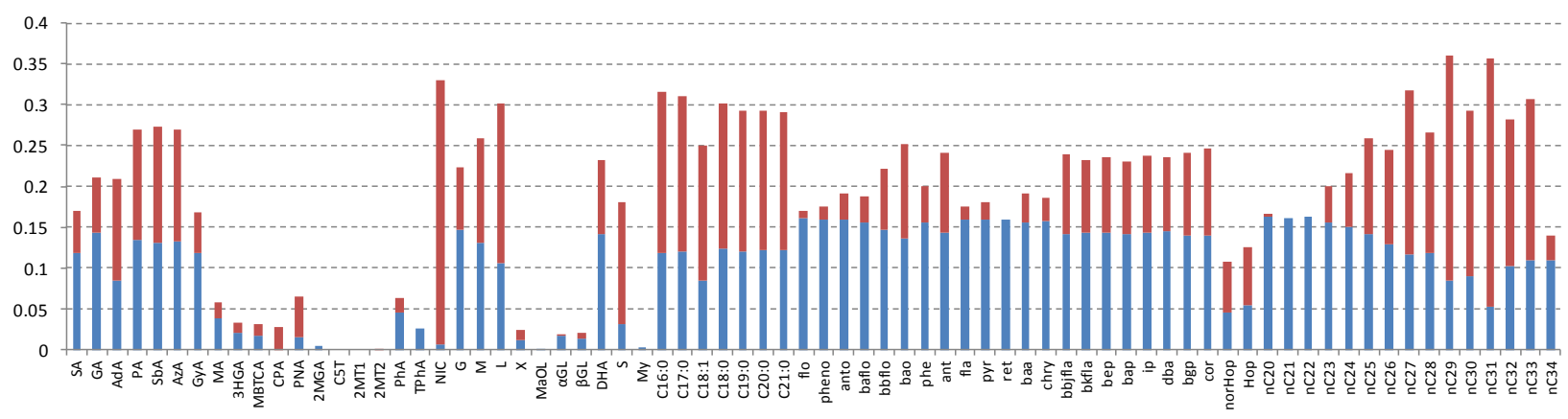

2)

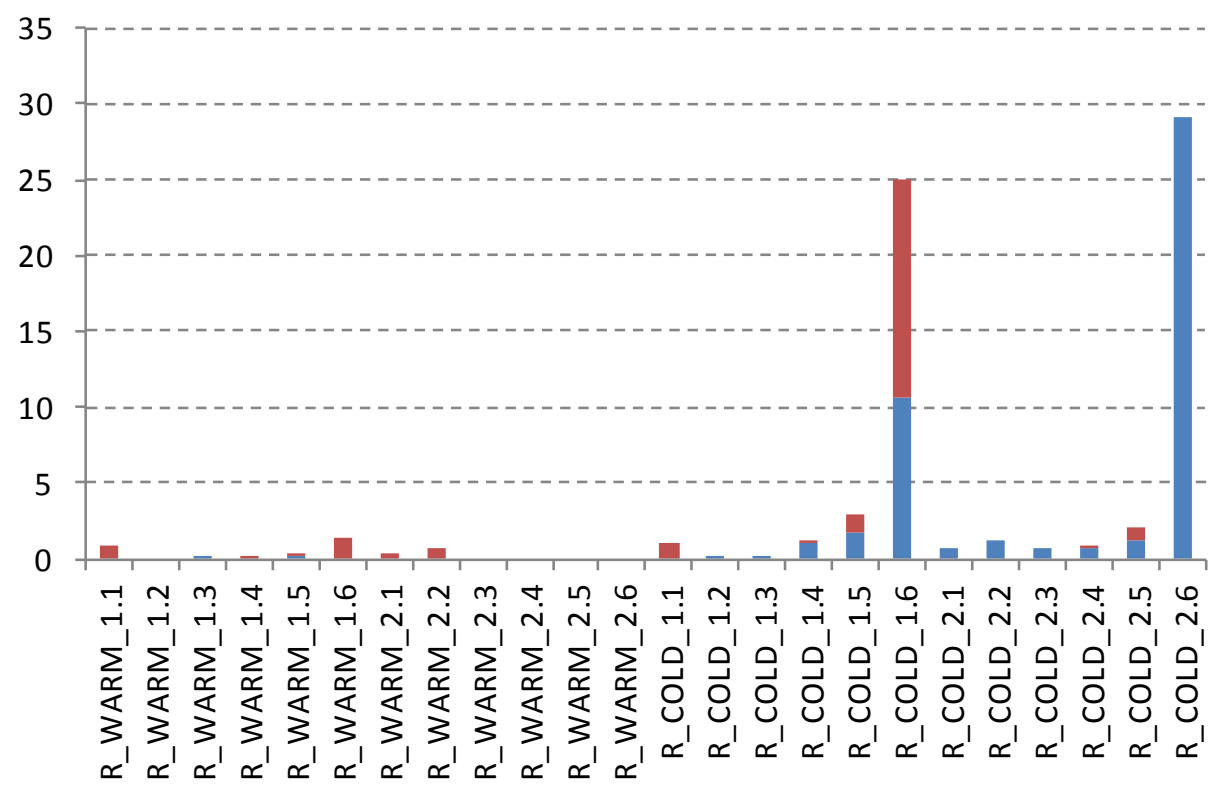

